\title{
X-ray structures of two proteins belonging to Pfam DUFI78 revealed unexpected structural similarity to the DUFI9I Pfam family
}

\author{
Rajiv Tyagi ${ }^{1}$, Stephen K Burley ${ }^{2}$ and Subramanyam Swaminathan*1
}

Address: ${ }^{1 B i o l o g y}$ Department, Brookhaven National Laboratory, Upton, New York 11973, USA and 2SGX Pharmaceuticals, Inc., San Diego, California 92121, USA

Email: Rajiv Tyagi - rtyagi@bnl.gov; Stephen K Burley - sburley@sgxpharma.com; Subramanyam Swaminathan* - swami@bnl.gov

* Corresponding author

Published: I October 2007

BMC Structural Biology 2007, 7:62 doi:10.1186/1472-6807-7-62

This article is available from: http://www.biomedcentral.com/l472-6807/7/62

(c) 2007 Tyagi et al; licensee BioMed Central Ltd.

This is an Open Access article distributed under the terms of the Creative Commons Attribution License (http://creativecommons.org/licenses/by/2.0), which permits unrestricted use, distribution, and reproduction in any medium, provided the original work is properly cited.
Accepted: I October 2007
Received: 3I May 2007

\begin{abstract}
Background: Pfam is a comprehensive collection of protein domains and families, with a range of well-established information including genome annotation. Pfam has two large series of functionally uncharacterized families, known as Domains of Unknown Function (DUFs) and Uncharacterized Protein Families (UPFs).

Results: Crystal structures of two proteins from Deinococcus radiodurans and Streptomyces coelicolor belonging to Pfam protein family DUFI78 (ID: PF0262I) have been determined using Selenium-Single-wavelength Anomalous Dispersion (Se-SAD). Based on the structure, we have identified the putative function for this family of protein.

Conclusion: Unexpectedly, we found that DUFI78 Pfam is remarkably similar to Pfam family DUFI9I suggesting that the sequence-based classification alone may not be sufficient to classify proteins into Pfam families.
\end{abstract}

\section{Background}

Pfam is a comprehensive collection of protein domains and families, with a range of well-established information including genome annotation. Each family in Pfam is represented by two multiple sequence alignments and two profile-Hidden Markov Models (profile-HMMs) [1]. Pfam has two large series of functionally uncharacterized families, known as Domains of Unknown Function (DUFs) and Uncharacterized Protein Families (UPFs). DUFs are families that have been created by Pfam whereas UPFs are those created by Swiss-Prot and added to Pfam [1]. The Protein Structure Initiative-2 has undertaken the task of structurally characterizing all Pfam families that have no structural representation. The Pfam protein family
DUF178 (ID: PF02621) consists of 61 proteins of unknown function, 59 from bacteria and 2 from archaebacteria [2]. Herein, we report the first crystal structures of DUF178 family members, including Q9RXE3 from Deinococcus radiodurans and Q9L0T8 Streptomyces coelicolor and show that they are remarkably similar to Pfam family DUF191.

\section{Results and discussion}

The structure of $10093 \mathrm{~b}$ was determined to $2.5 \AA$ resolution using Selenium-SAD (Table 1). The final refined model of $10093 \mathrm{~b}$ contains 8 protomers, 18 sulfate ions, and 653 water molecules. The final refined model of $10093 \mathrm{f}(2.04 \AA$ resolution) contains 4 protomers and 579 
water molecules. Despite low sequence identity (27\%; Figure 1), the polypeptide chain structures are very similar (Figure 2). The two structures superimpose well with a rmsd of $1.9 \AA$ for $252 \alpha$-carbon atomic pairs, excluding a loop region between Val166 to Ser177 in 10093b that does not occur in 10093f (Figure 3A). This region is absent in all other sequences shown in Figure 1, except in Thermus thermophilus (Figure 1).

Notwithstanding the similarity of the two polypeptide chain folds, MR attempts with various 10093b-derived search models were not successful. Molecular replacement may have failed because of low sequence identity and/or due to the presence of multiple protomers within the asymmetric unit.

\section{Biologically active units 10093b}

Eight monomers A, B, C, D, E, F, G, and $\mathrm{H}$ found in the asymmetric unit form four tightly packed dimers $(A B, C D$, $\mathrm{EF}$, and $\mathrm{GH}$ ). Each dimer pair superimposes very well on the remaining three with rmsds $=0.74 \AA-0.78 \AA$ for all $\alpha$ carbon atomic pairs. A dimer interface analysis performed using PDBSUM [3] demonstrated that each pair buries $\sim 3,000 \AA^{2}$ of solvent accessible surface area $(12.3 \%$ of the total area), a value higher than would be found typically in interacting surfaces for a protein of this size [4]. At least 26 residues from each half of the dimer participate in intermolecular interactions. There are 6 direct hydrogen bonded interactions between the protein atoms across the dimer interface. Such a tight dimer interface suggests that the $10093 \mathrm{~b}$ dimer is functional, which is supported by the results of analytical gel filtration (data not shown).

\section{$10093 f$}

Four monomers A, B, C, and D found in the crystallographic asymmetric unit occur as two dimers $(A B$ and $\mathrm{CD})$, albeit with an intermolecular packing arrangement that differs from that seen for 10093b. Each observed 10093f dimer pair buries $\sim 1800 \AA^{2}$ of solvent accessible surface area, which is not thought to be significant for a protein of this size. Moreover, the gel-filtration analysis

Table I: Data collection, phasing and refinement statistics.

\begin{tabular}{|c|c|c|}
\hline & Se -SAD (1 0093b) & Se -SAD (10093f) \\
\hline Cell dimensions & $a=75.3, b=139.4, c=153.6 ; \beta=92.8^{\circ}$ & $a=75.8, b=97.4, c=86.6 ; \beta=106.5^{\circ}$ \\
\hline Space group & $\mathrm{P} 2_{1}$ & $\mathrm{P} 2_{1}$ \\
\hline \multicolumn{3}{|l|}{ Data Collection Statistics } \\
\hline Wavelength $(\AA)$ & 0.98 & 0.98 \\
\hline Temperature $(\mathrm{K})$ & 100 & 100 \\
\hline Resolution range & $50.0-2.5$ & $50-2.04$ \\
\hline Outermost Shell $(\AA ̊)$ & $2.59-2.5$ & $2.11-2.04$ \\
\hline Unique reflections & $107665(9866)$ & $76032(6908)$ \\
\hline Completeness (\%) & $98.9(91.0)$ & $98.8(89.7)$ \\
\hline Mean $I / \sigma(I)$ & $11.1(1.9)$ & $16.8(2.1)$ \\
\hline Redundancy & $6.8(5.2)$ & $4.6(3.9)$ \\
\hline $\begin{array}{l}R_{\text {merge }} \mathrm{I} \\
\text { Phasing Statistics }\end{array}$ & $0.095(0.40)$ & $0.043(0.21)$ \\
\hline Phasing power ${ }^{2}$ (ano) & 0.83 & 0.94 \\
\hline FOM3: & 0.29 & 0.27 \\
\hline After density modification & 0.93 & 0.92 \\
\hline \multicolumn{3}{|l|}{ Refinement Statistics } \\
\hline No. of reflections (work) & 100602 & 71996 \\
\hline No. of reflections (test) & 3150 & 2264 \\
\hline${ }^{4} R_{\text {factor }} / 5 R_{\text {free }}$ & $0.20 / 23.4$ & $0.24 / 0.28$ \\
\hline Resolution range $(\AA)$ & $50.0-2.5$ & $30.0-2.04$ \\
\hline RMSD for bond length $(\AA)$ & 0.006 & 0.006 \\
\hline RMSD bond angles $\left({ }^{\circ}\right)$ & 1.35 & 1.4 \\
\hline \multicolumn{3}{|l|}{ <B-values> } \\
\hline Main-chain $\left(\AA^{2}\right)$ & 30.8 & 30 \\
\hline Side-chain $\left(\AA^{2}\right)^{\prime}$ & 32.4 & 32.4 \\
\hline \multicolumn{3}{|l|}{ Number of non-H atoms } \\
\hline No. of heteroatoms & 90 & 0 \\
\hline No of water molecules & 653 & 579 \\
\hline
\end{tabular}

Values for the highest resolution shell are given within parentheses.

${ }^{\prime} R_{\text {merge }}=\left.\Sigma\left|I_{i}-\langle 1\rangle / \Sigma\right|\right|_{i} \mid$ where $I_{i}$ is the intensity of the $i^{\text {th }}$ measurement, and $\langle I\rangle$ is the mean intensity for that reflection. 2Phasing power and ${ }^{3} \mathrm{FOM}$ (Figure of merit) as defined in SHARP.

${ }^{4} \mathrm{R}_{\text {factor }}=\Sigma|| \mathrm{F}_{\text {obs }}|-| \mathrm{F}_{\text {calc }}|| / \Sigma\left|\mathrm{F}_{\text {obs }}\right|$ where $\left|\mathrm{F}_{\text {calc }}\right|$ and $\left|\mathrm{F}_{\text {obs }}\right|$ are the calculated and observed structure factor amplitudes, respectively. 

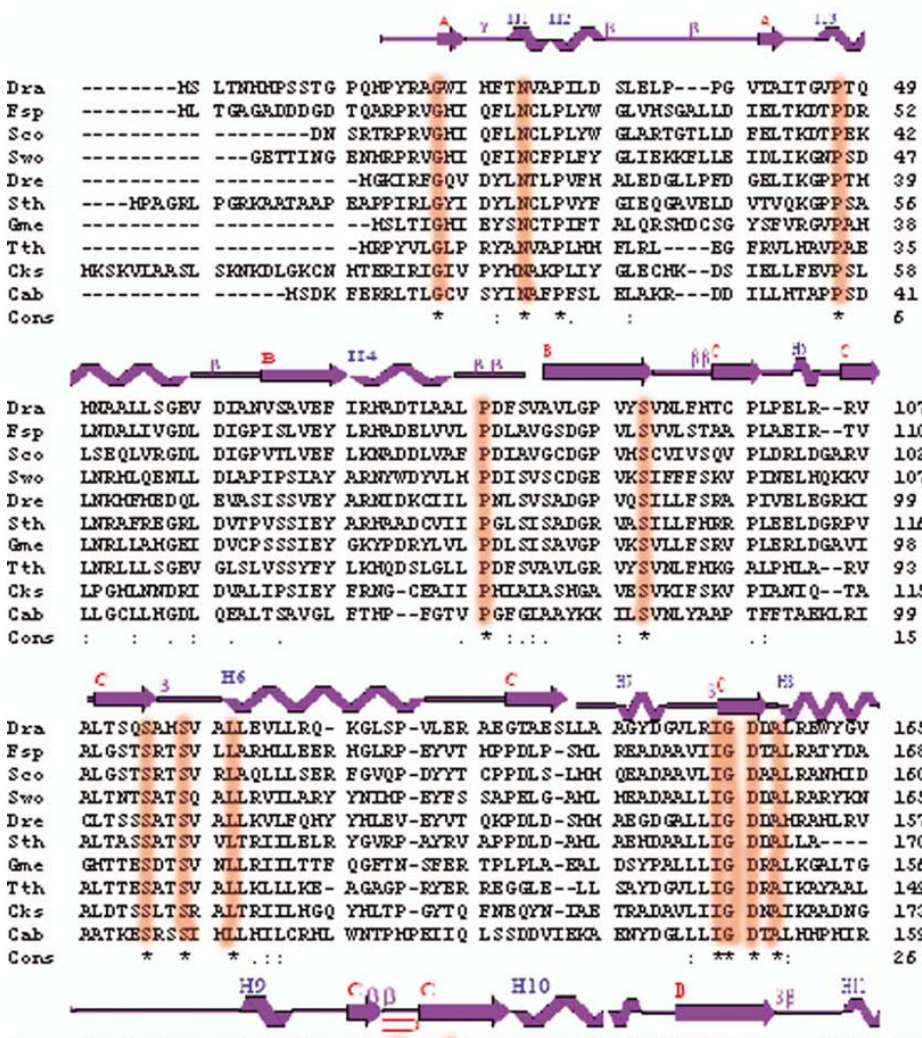

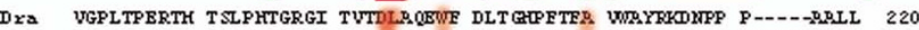

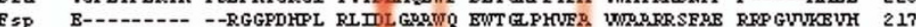

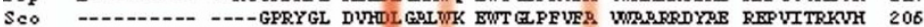
SWO ---1. Dre Sth - 217 Ghe -

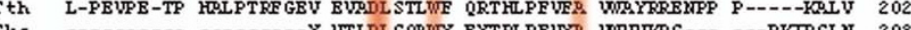
Cks -

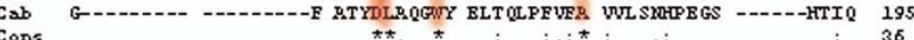
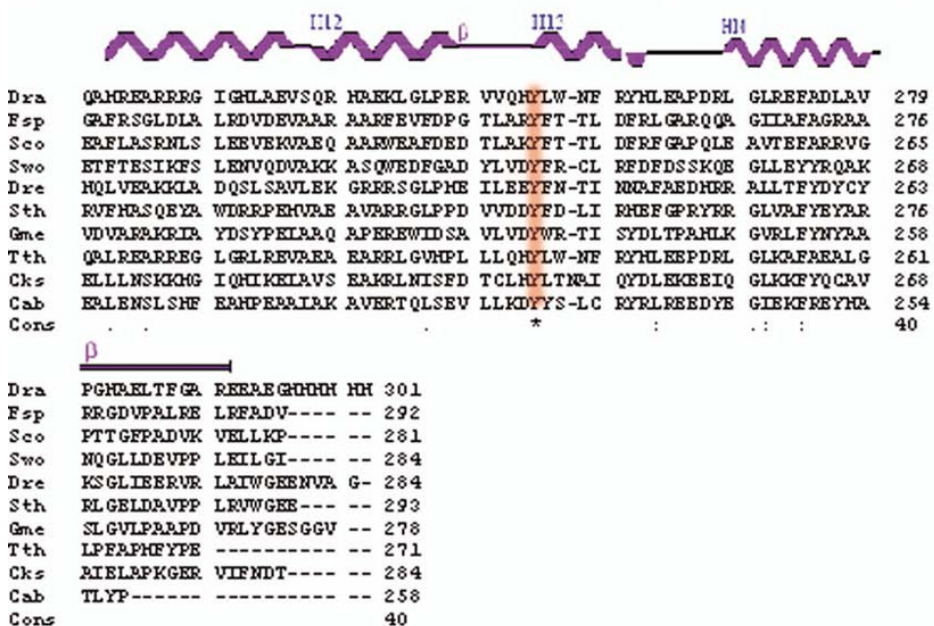

\section{Figure I}

Multiple sequence alignment of DUFI 78 from various organisms. The residues highlighted in orange are the conserved residues (*). The abbreviations for organism names are as follows: Dra; Deinococcus radiodurans (I0093b), Fsp; Frankia sp. Ccl3, Sco; Streptomyces coelicolor ( $10093 f$ ), Dre; Desulfotomaculum reducens, Sth; Symbiobacterium thermophilum, Gme; Geobacter metallireducens, Tth; Thermus thermophilus, Cks; Candidatus Kuenenia stuttgarti, Cab; Chlamydophila abortus. The secondary structural elements for 10093b (residues 16 to 285) are given at the top of alignment. 


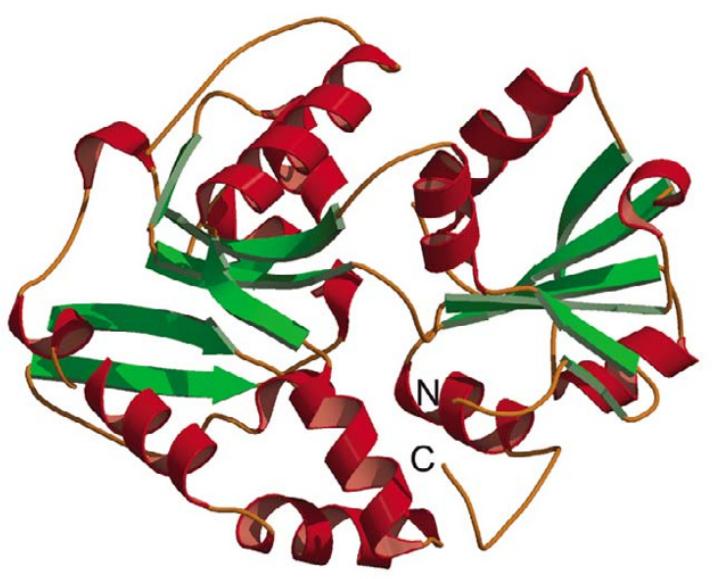

(A)

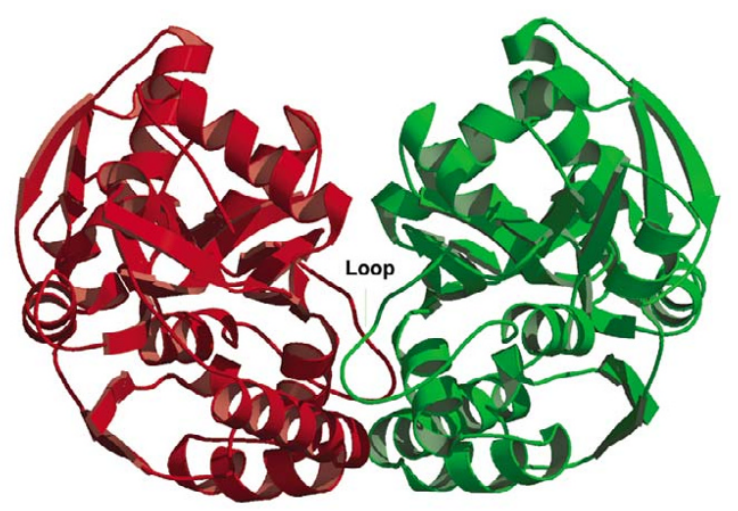

(B)

\section{Figure 2}

(A) Monomer of $10093 \mathrm{~b}$ showing both N- \& C-terminal domains. The $10093 \mathrm{f}$ monomer has the same fold. (B) Asymmetric unit of $10093 \mathrm{~b}$ showing tightly packed dimer. Loop responsible for dimerization is labeled.

(data not shown) revealed that $10093 \mathrm{f}$ is a monomer in solution.

In the $10093 \mathrm{~b}$ dimer, Glu172 from the atypical Val166Ser177 loop makes one of the six hydrogen bonds present in the dimer interface while three other residues in the same loop contribute to van der Waals interactions. We suggest that the atypical loop found in 10093b may help support dimerization. The absence of this loop in $10093 \mathrm{f}$ may explain the monomeric solution behavior of this family member.

\section{Structure and sequence relationships and homology modeling}

In an effort to annotate the function of these two related proteins, bioinformatics analysis with the experimental structures was performed using DALI [5]. For 10093b, a DALI search revealed only two structural matches with a Z-score greater than 10 . The closest match, AF1704 from Archaeoglobus fulgidus (PDB ID: 1ZBM), a protein of unknown function belonging to Pfam DUF191 gave a Zscore of 19.8 with sequence identity of $15 \%$ and rmsd of $2.7 \AA$ between 227 structurally equivalent $\alpha$-carbon atomic pairs. Thus, structure determination of $10093 \mathrm{~b}$ not only provided the first structural information for the Pfam family DUF178, it also documented unexpected structural similarity to a member of the DUF191 Pfam family that could not have been reliably predicted from sequence comparisons alone. A DALI search with $10093 \mathrm{f}$ revealed (excluding 10093b) significant matches (i.e., Z-score greater than 10) with AF1704 Z-score 19.4, as expected, and with a nitrate transport protein (PDB ID: 2G29) Zscore of 17.8 with sequence identity of $12 \%$ and rmsd between 252 structurally equivalent $\alpha$-carbon atomic pairs of $3.3 \AA$ A. Stereoviews of the superimposed polypeptide chains of $10093 \mathrm{~b}$ over $10093 \mathrm{f}$-monomers, -dimers and $10093 \mathrm{~b}$ over $1 \mathrm{ZBM}$ are presented in Figure 3A, 3B and $3 \mathrm{C}$ respectively.

A BLAST [6] search of Uniprot protein sequence database using the sequences of both $10093 \mathrm{~b}$ and $10093 \mathrm{f}$ yielded 61 matches (sequence identities $=83-27 \%$ ). Virtually all of the matches are identified as bacterial or archaeal hypothetical proteins. The three exceptions are as follows: a SAM-dependent methyltransferase from Lactococcus lactis (34\% identity), leucyl-tRNA synthetase from Xanthomonas campestris (28\% identity), D-alanine-D-alanine ligase from Nitrosomonas europaea (27\% identity).

At the time of publication, the experimental structures of $10093 \mathrm{~b}$ and $10093 \mathrm{f}$ were used as a template to compute homology models of 1133 proteins with related sequences with the Modweb server [7]. The 17 models out of 1133 had sequence identity of great than $30 \%$.

\section{Active site/ligand binding site prediction}

Active site/ligand binding site prediction performed using CASTp [8] revealed two major clefts on the surfaces of the $10093 \mathrm{~b}$ and $10093 \mathrm{f}$ (10093b estimated areas: $699.3 \AA 2$ and $277 \AA^{2}$ ). Further analysis of these surface features together with a multiple sequence alignment performed in ClustalW [9] and edited in BioEdit [10] (Figure 1) performed for $10093 \mathrm{~b}$ reveals the presence of most of the conserved residues, including Asn26, Pro47, Ser92, Ser113, Ser116, Ile154, Gly155, and Asp156 (Figure 4). We suggest that this larger cleft represents the active site 

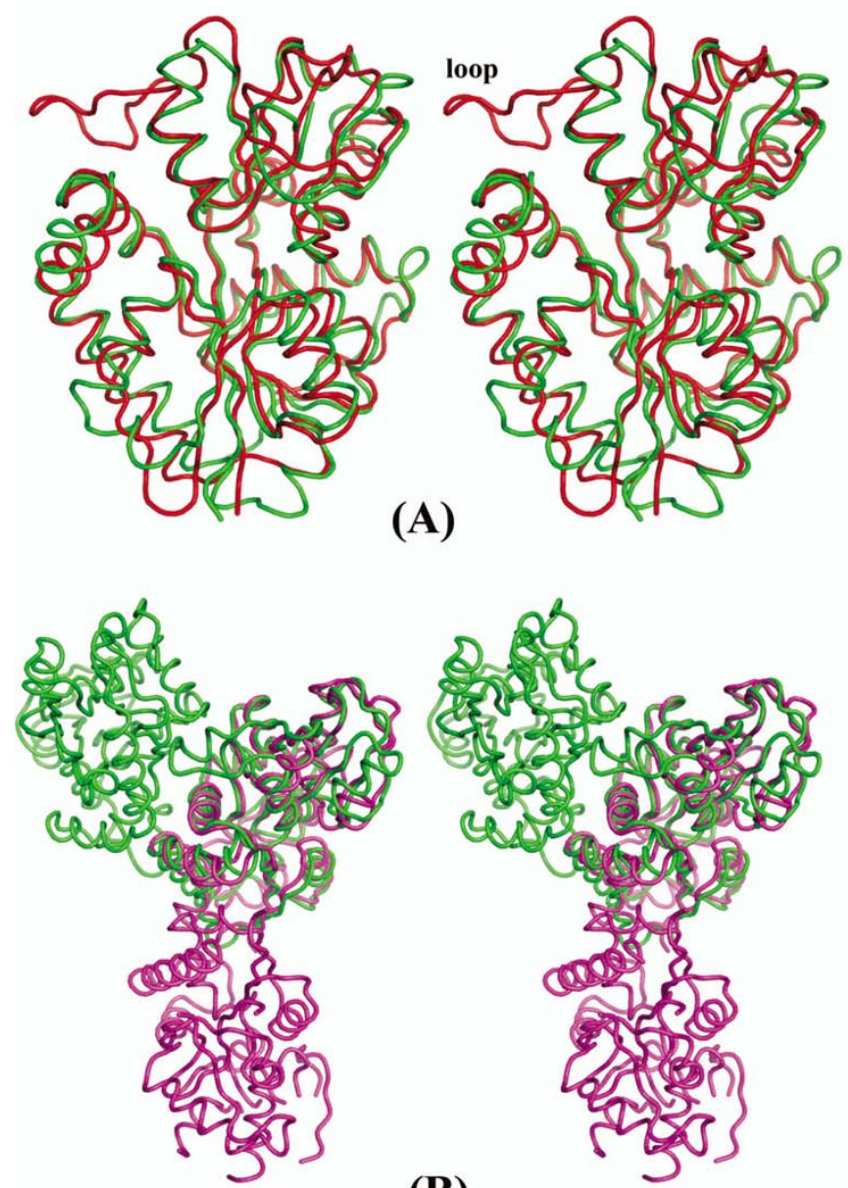

(B)
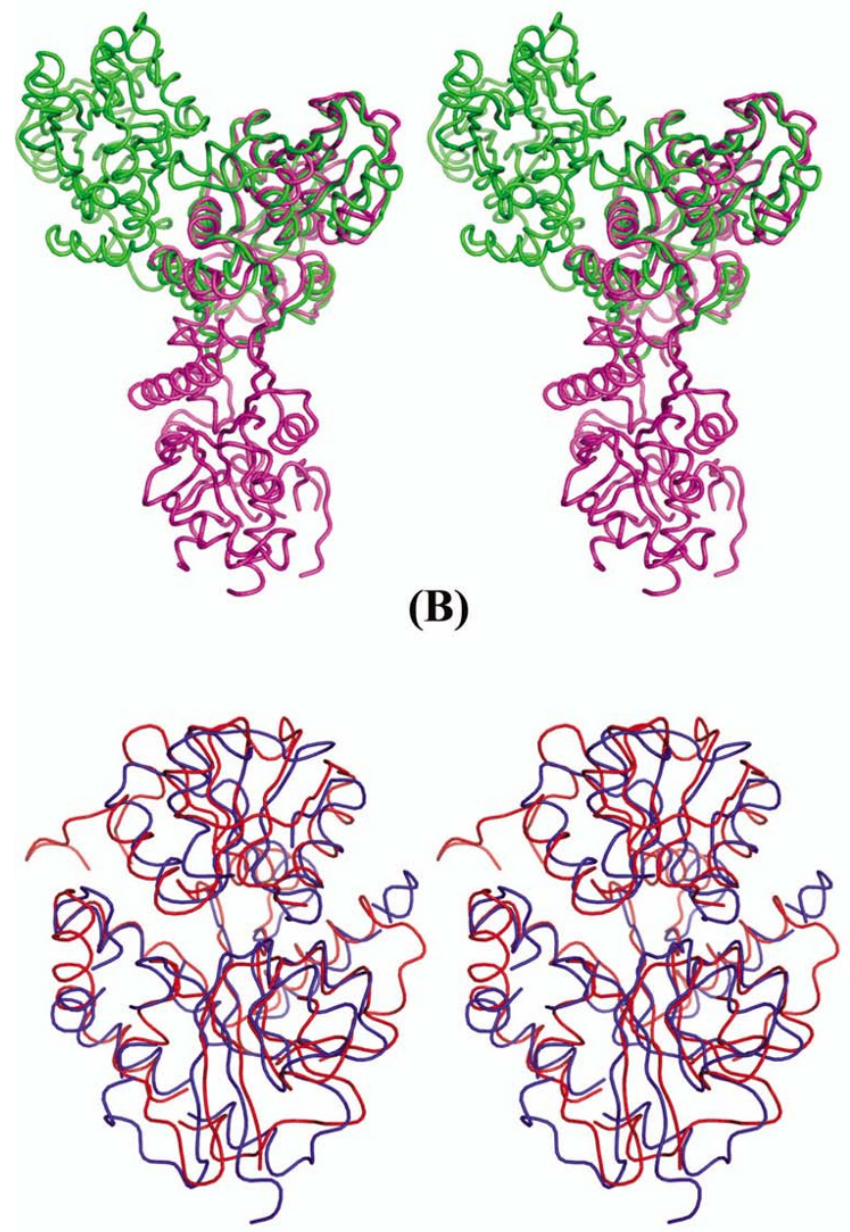

(C)

\section{Figure 3}

Stereoviews of (A) superposition of $10093 \mathrm{~b}$ (red) and $10093 \mathrm{f}$ (green) monomers. The loop exclusive to $10093 \mathrm{~b}$ is labeled. (B) superposition of $10093 \mathrm{~b}$ (green) and $10093 \mathrm{f}$ (magenta) dimer. While $10093 \mathrm{~b}$ is a dimer in solution, the dimerization of $10093 \mathrm{f}$ is due to crystal packing effect. (C) superposition of I0093b (red) and IZBM (blue) monomers.

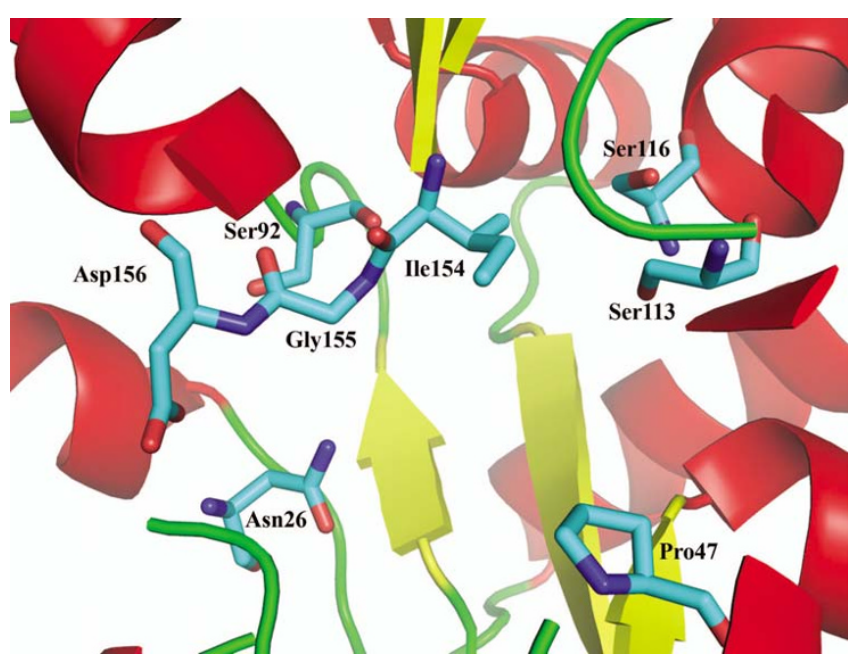

Figure 4

Zoom view showing putative binding site of $10093 \mathrm{~b}$. The $\beta$ strands (yellow ribbon), $\alpha$-helices (red ribbon), and random coil (green ribbon), plus clustered conserved residues Asn26, Pro47, Ser92, SerI I3, Ser I 16, Ile I54, Gly I55, and Asp I 56 from multiple sequence alignment shown in figure I (atom color coded stick figures; N-blue; C-sky blue; O-red). All residues numbers are labelled in black.

and/or ligand binding site for this functionally uncharacterized Pfam family.

\section{Prediction of putative function}

$10093 \mathrm{~b}$ and $10093 \mathrm{f}$ belonging to DUF178 family have remarkable similarity to DUF191 protein family and a nitrate-binding protein (2G29). This similarity was not evident from sequence comparison alone. Moreover, the uncharacterized DUF191 family of proteins is annotated to be putative solute-binding protein. Our analysis of the surface clefts shows that the major cleft identified in $10093 \mathrm{~b}$ is common to all of them, the size being nearly the same. The binding pocket of 10093b superposes well with that of nitrate-binding protein. Further analysis of the active site shows that the entry of this cavity is occupied by hydrophobic residues as in 2G29 [11]. In 10093b, the residual density in the difference Fourier showed a dumb bell shaped density and was modeled as water. This could very well be an unidentified ion. Based on the comparison of structures and binding sites, we predict that this protein could be a solute binding protein, though we cannot at this stage identify the nature of the ion. The weak homology implies that it may be a different kind of solute.

\section{Conclusion}

The structure determination of 10093b and 10093f has revealed the unexpected similarity between DUF178 and DUF191 family of proteins suggesting that the information from three-dimensional structures along with the 
sequence information will predict the family of proteins of similar functions more reliably. We have predicted the protein to be a solute-binding protein based on structure and binding cavity. Fold and structural similarity among proteins with low sequence identity (less than 30\%) is not uncommon. One prominent example would be proteins classified into different Pfams but with the same TIM barrel fold [12]. Amidohydrolases with low sequence homology have various substrate specificities and different enzymatic functions but all of them have similar fold. These belong to different Pfam families but are grouped together as a super family [12]. This also suggests that DUF178 and DUF191, though belonging to different Pfams of unknown functions, may be members of the same superfamily.

\section{Methods \\ Protein production $10093 b$}

The target gene for 10093b was amplified using polymerase chain reaction (PCR) from Deinococcus radiodurans genomic DNA using a forward (ACCAACCATCACCCATCATCTAC) and a reverse (CTGCTTCCTCACGCGCTCCGAAG) primer.

\section{$10093 f$}

The target gene was amplified similar to 10093b from Streptomyces coelicolor genomic DNA using Forward (GATAATAGCCGTACCCGCCC) and a reverse (CAGGTTTCAGCAACTCAACCTTG) primer.

The amplified genes of both 10093b and 10093f were gel purified and cloned into pSGX3 (BC) vector designed to express the protein of interest with a C-terminal hexa-histidine tag to facilitate easy and high yield purification. Protein expression/purification utilized previously published protocols [13]. For 10093b a yield of $22 \mathrm{mg}$ was obtained from 3L culture, whereas for $10093 \mathrm{f}$ the yield was $91 \mathrm{mg}$ from $2 \mathrm{~L}$ culture.

\section{Crystallization, data collection and structure determination $10093 b$}

Native and Se-Met crystals of $10093 \mathrm{~b}$ were grown at $20^{\circ} \mathrm{C}$ via the sitting drop vapor diffusion method (crystallization drop contained $2 \mu \mathrm{L}$ of $22 \mathrm{mg} / \mathrm{mL}$ protein plus $2 \mu \mathrm{L}$ of reservoir solution containing 25\% (w/v) PEG 3350, 0.1 $\mathrm{M}$ Bis-Tris pH 5.5, 0.2 $\mathrm{M} \mathrm{NH}_{4} \mathrm{SO}_{4}$, and $1 \mu \mathrm{L}$ of $0.1 \mathrm{M}$ TCEP hydrochloride). Rod shaped crystals with dimensions 0.5 $\times 0.02 \times 0.02 \mathrm{~mm}^{3}$ appeared after two days. Crystals were flash frozen in liquid nitrogen following addition of $20 \%$ ethylene glycol to the mother liquor. Diffraction data were collected at beamline X12C, National Synchrotron Light Source (NSLS), Brookhaven National Laboratory and processed using HKL2000 [14]. Both crystals belong to monoclinic space group $\mathrm{P} 2{ }_{1}$. The calculated Matthews coefficient is $3.1 \AA 3 / \mathrm{Da}$ (solvent content $59.4 \%$ by volume), assuming eight molecules/asymmetric unit. All 32 possible selenium sites were found by SHELXD [15] using the peak data collected at the selenium absorption edge $(\lambda$ $=0.98 \AA$ ). Phase refinement and density modification were performed with SHARP [16]. The final improved electron density map after density modification was of high quality and allowed automated model building of about $85 \%$ of the polypeptide chain with ARP/wARP [17]. The remainder of the polypeptide chain was built manually using both Sigma-weighted $2|\mathrm{Fo}|-|\mathrm{FC}|$ difference Fourier map from CNS and experimental electron density map from SHARP using O [18]. The structural model was refined to convergence using CNS [19]. For $\mathrm{R}_{\text {free }}$ calculation $3 \%$ of randomly selected data was excluded from the refinement. The Ramachandran plot calculated using PROCHECK [20], shows $89.9 \%$ residues in the most favorable region. Arg173 in chain $\mathrm{G}$ in the loop region occurs in disallowed region, probably because of poor resolution of the electron density. The structures of individual $10093 \mathrm{~b}$ protomers found in the asymmetric unit were highly similar to one another (pairwise root-meansquare-deviations or rmsds $=0.74 \AA-0.78 \AA$ ).

\section{$10093 f$}

Rod shaped crystals (dimensions; $0.3 \times 0.02 \times 0.02 \mathrm{~mm}^{3}$ ) similar to $10093 \mathrm{~b}$ were obtained for native protein in 25\% (w/v) PEG 3350, 0.1 M Bis-Tris pH 5.5, and 0.2 M $\mathrm{MgCl}_{2}$ and $10 \%$ Jeffamine and native diffraction data were collected at beamline X12C. As sequence identity with $10093 \mathrm{~b}$ was $27 \%$, molecular replacement (MR) was attempted but did not yield meaningful phases. Accordingly, SeMet protein was crystallized using similar condition and Se-SAD diffraction data were collected at beamline X29A (NSLS). 10093f crystals grow in monoclinic P2 1 space group with four molecules/asymmetric unit. All possible 16 selenium sites were found by SHELXD [15]. Phase refinement and density modification were performed in SHARP [16]. The final improved electron density map after density modification was of high quality and allowed automated model building of about $85 \%$ of the polypeptide chain with ARP/wARP [17]. The remainder of the polypeptide chain was built manually using $O$ [18], and the resulting structural model was refined to convergence using CNS [19]. The Ramachandran plot calculated using PROCHECK [20] shows $89.1 \%$ residues in the most favorable region. Five residues (Ala146 and Met 84 in chain A, Met84 in chain B, Met84 and Leu136 in chain C and Met84 in chain D) occur in disallowed region, probably because of poor resolution of the electron density. The structures of individual $10093 \mathrm{f}$ protomers found in the asymmetric unit were highly similar to one another (pairwise rmsds $=0.76-0.78 \AA$ ). 
Data collection, phasing and refinement statistics for both structures are provided in Table 1. The coordinates and structure factors of both structures have been deposited with the Protein Data Bank (10093b: 2I6E; 10093f: $2 \mathrm{NXO})$.

\section{Competing interests}

The author(s) declares that there are no competing interests.

\section{Authors' contributions}

RT carried out crystallographic studies and prepared the manuscript. SKB helped in preparing the manuscript. SS helped in determining and analyzing the structure.

\section{Acknowledgements}

Research was supported by a U54 award from the National Institute of General Medical Sciences to the NYSGXRC (GM074945; PI: Stephen K. Burley) under DOE Prime Contract No. DEAC02-98CHI 0886 with Brookhaven National Laboratory. We would also like to thank the PXRR and Dr Saxena at National Synchrotron Light Source for their co-operation during data collection. We are grateful to Drs. Eswaramoorthy and Kumaran for helpful discussions.

\section{References}

I. Bateman A, Coin L, Durbin R, Finn RD, Hollich V, Griffiths-Jones S, Khanna A, Marshall M, Moxon S, Sonnhammer ELL, Studholme D], Yeats C, Eddy SR: The Pfam protein families database. Nucleic Acids Research 2004, 32:DI38-D I4I.

2. Camon E, Magrane M, Barrell B, Binns D, Fleischmann W, Kersey P, Mulder N, Oinn T, Maslen J, Cox A, al : The Gene Ontology Annotation (GOA) Project: Implementation of GO in SWISSPROT, TrEMBL, and InterPro. Genome Res 2003, 13:662-672.

3. Laskowski RA: PDBsum: summaries and analyses of PDB structures. Nucleic Acids Res 2001, 29:221-222.

$4 . \quad J o n e s \mathrm{~S}$, Thornton JM: Principles of protein-protein interactions. Proc Natl Acad Sci U S A 1996, 93:13-20.

5. Holm L, Sander C: Protein structure comparison by alignment of distance matrices. J Mol Biol 1993, 233:123-138.

6. Altschul SF, Madden TL, Schaffer AA, Zhang J, Zhang Z, Miller W, Lipman D]: Gapped BLAST and PSI-BLAST: a new generation of protein database search programs. Nucleic Acids Res 1997, 25:3389-3402.

7. Eswar N, John B, Mirkovic N, Fiser A, llyin VA, Pieper U, Stuart AC, Marti-Renom MA, Madhusudhan MS, Yerkovich B, Sali A: "Tools for comparative protein structure modeling and analysis." . Nucleic Acids Res 2003, 31:3375-3380.

8. Binkowski TA, Naghibzadeh S, Liang J: CASTp: Computed Atlas of Surface Topography of proteins. Nucleic Acids Research 2003, 3I: 3352-3355.

9. Thompson JD, Higgins DG, Gibson TJ: CLUSTAL W: improving the sensitivity of progressive multiple sequence alignment through sequence weighting, position-specific gap penalties and weight matrix choice. Nucleic Acids Res 1994, 22:4673-4680.

10. Hall T: BioEdit. Biological sequence alignment editor for Windows. 1998 [http://www.mbio.ncsu.edu/BioEdit/bioedit.html]. NC, North Carolina State University

II. Koropatkin NM, Pakrasi HB, Smith TJ: Atomic structure of a nitrate-binding protein crucial for photosynthetic productivity. Proc Natl Acad Sci U S A 1 03:9820-9825.

12. Boeckmann B, Bairoch A, Apweiler R, Blatter MC, Estreicher A, Gasteiger E, Martin MJ, Michoud K, O'Donovan C, Phan I, Pilbout S, Schneider M: The SWISS-PROT protein knowledgebase and its supplement TrEMBL. Nucleic Acids Res 2003, 31:365-370.

13. Rao KN, Bonanno JB, Burley SK, Swaminathan S: Crystal Structure of Glycerophosphodiester Phosphodiesterase From Agrobacterium tumefaciens by SAD With a Large Asymmetric Unit. Proteins: Structure, function, and Bioinformatics 2006, 65:5 I4-518.
14. Otwinowski Z, Minor W: "Processing of X-ray Diffraction Data Collected in Oscillation Mode". Methods Enzymol 1997, 276:307-326.

15. Uson I, Sheldrick GM: Advances in direct methods for protein crystallography. Curr Op Struct Biol 1999, 9:643-648.

16. Fortelle EDL, Bricogne G: Maximum-likelihood heavy atom parameter refinement in the MIR and MAD methods. Methods Enzymol 1997, 276:472-493.

17. Perrakis A, Morris R, Lamzin VS: Automated protein model building combined iterative structure refinement. Nat Struct Biol 1999, 6:458-463.

18. Jones TA, Zou JY, Cowan SW, Kjeldgaard M: Improved methods for the building of protein models in electron density maps and the location of errors in these models. Acta Crystallogr I99I, A47:II0-II9.

19. Brunger AT, Adams PD, Clore GM, DeLano WL, Gros P, GrosseKunstleve RW, Jiang JS, Kuszewski J, Nilges M, Pannu NS, Read RJ, Rice LM, Simonson T, Warren GL: Crystallography \& NMR system: A new software suite for macromolecular structure determination. Acta Crystallogr D Biol Crystallogr 1998, 54:905-92I.

20. Laskowski RA, MacArthur MW, Moss DS, Thornton JM: PROCHECK: a program to check the stereochemical quality of protein structures. J Appl Cryst 1993, 26:283-29I.
Publish with Biomed Central and every scientist can read your work free of charge

"BioMed Central will be the most significant development for disseminating the results of biomedical research in our lifetime. "

Sir Paul Nurse, Cancer Research UK

Your research papers will be:

- available free of charge to the entire biomedical community

- peer reviewed and published immediately upon acceptance

- cited in PubMed and archived on PubMed Central

- yours - you keep the copyright 